\title{
INTEGRALLY CLOSED MODULES OVER TWO-DIMENSIONAL REGULAR LOCAL RINGS
}

\author{
VIJAY KODIYALAM
}

\begin{abstract}
This paper is based on work of Rees on integral closures of modules and initiates the study of integrally closed modules over two-dimensional regular local rings in analogy with the classical theory of complete ideals of Zariski. The main results can be regarded as generalizations of Zariski's product theorem. They assert that the tensor product mod torsion of integrally closed modules is integrally closed, that the symmetric algebra mod torsion of an integrally closed module is a normal domain and that the first Fitting ideal of an integrally closed module is an integrally closed ideal. A construction of indecomposable integrally closed modules is also given. The primary technical tool is a study of the Buchsbaum-Rim multiplicity.
\end{abstract}

\section{INTRODUCTION}

The theory of complete ideals in two-dimensional regular local rings is rich both in structural and numerical results. The principal structural results are the product theorem and the unique factorization theorem of Zariski ([ZrsSml, Appendix 5]) which assert that the complete ideals are closed under the product operation and have unique factorization into simple complete ideals. Much work has gone into trying to understand and generalize these results both to higher dimensions and weakening the hypothesis of regularity. See for instance the papers [Ghn], [Lpm], [HnkSll], [Ctk2] and their bibliographies. Examples due to Cutkosky ([Ctk]), Huneke ([Hnk2]) and Lipman ([Lpm]) show that the main results of Zariski are false even in regular local rings of dimension three.

This paper generalizes Zariski's product theorem in a different direction. The notions of integral closures and reductions of torsion-free modules over Noetherian domains have recently been intoduced by Rees ([Rs]). A natural product on the torsion-free modules is tensor product modulo torsion which in the case of ideals reduces to the ordinary product. It is then natural to ask what parts of Zariski's theory may be extended to finitely generated integrally closed modules over two-dimensional regular local rings.

It turns out that a substantial portion of Zariski's theory generalizes. The main theorems of this paper are a first step in such an extension. Precisely, we prove first that over a two-dimensional regular local ring, the product of integrally closed modules is itself integrally closed. We also show that the torsionfree symmetric algebra of an integrally closed module is a normal domain. Fi-

Received by the editors October 10, 1994.

1991 Mathematics Subject Classification. Primary 13B21, 13B22, 13H05, 13 H 15. 
nally, we show that a certain Fitting ideal of an integrally closed module is a complete ideal.

The structure of the proofs are patterned closely after the proof for ideals as in Huneke's expository paper [Hnk]. Thus the main ingredients are a numerical criterion for a module to be contracted and a theorem asserting the decrease of multiplicity on taking transforms. The appropriate notion of multiplicity here is the Buchsbaum-Rim multiplicity.

In Section 2 we define some numerical invariants for finitely generated torsion-free modules over two-dimensional regular local rings and establish some relationships that hold between these invariants. One of the main results is a numerical criterion for such a module to be contracted from a point on the blow-up of the maximal ideal of $R$. In Section 3 we discuss the definitions of reductions and integral closures of modules and the related notion of Buchsbaum-Rim multiplicity. This section is basically a collection of various results of Rees ([Rs]) and Buchsbaum-Rim ([BchRm]). The heart of the proof is contained in Section 4. The main result of this section is a proof that the Buchsbaum-Rim multiplicity decreases on taking transforms. Section 5 brings together the material from the previous sections to show that products, torsion-free symmetric powers and torsion-free exterior powers of integrally closed modules are all integrally closed. The non-triviality of the theory is shown by a construction and some examples.

\section{Contracted modules}

The main results of this section are contained in Propositions 2.2, 2.5 and in Theorem 2.8. Each of these is an analogue for torsion-free modules of a result known in the case of ideals. The proofs generalize those in [Hnk]. Just as contracted ideals play an important role in the theory of integrally closed ideals, so do contracted modules in the theory of integrally closed modules.

Throughout this section, $R$ will denote a two-dimensional regular local ring with maximal ideal $\mathbf{m}$, infinite residue field $k$ and field of fractions $K$. The valuation of $K$ determined by the powers of $\mathbf{m}$ is denoted $\operatorname{ord}_{R}(-)$. Therefore, for an element $a \in R$, by definition, $\operatorname{ord}_{R}(a)=$ largest integer $n$ such that $a \in \mathbf{m}^{n}$. Similarly, for an ideal $I$ of $R$, we set $\operatorname{ord}_{R}(I)=$ largest integer $n$ such that $I \subseteq \mathbf{m}^{n}$.

Let $(-)^{*}$ denote the functor $\operatorname{Hom}_{R}(-, R)$. In this section, $M$ always denotes a finitely generated torsion-free $R$-module and $F$ stands for its double dual, i.e., $F=M^{* *}$. We begin with the following proposition which is well-known and gives a characterization of $F$ :

Proposition 2.1. For a finitely generated torsion-free $R$-module $M$, the double dual $F$ is a free $R$-module which canonically contains $M$ and is such that the quotient module $F / M$ is of finite length. Moreover, if $G$ is any free $R$ module containing $M$ such that $G / M$ is of finite length, then there is a unique isomorphism $F \rightarrow G$ that restricts to the identity on $M$.

Proof. Direct calculation shows that over any domain $R$, a regular sequence of length 2 on $R$ is also a regular sequence on the dual of any $R$-module. Thus here, $\operatorname{depth}_{R}\left(M^{*}\right)=2$ and then it follows from the Auslander-Buchsbaum formula that $M^{*}$ is a free $R$-module. Hence, so is $F=M^{* *}$.

Since $M$ is a finitely generated torsion-free $R$-module, the natural map 
$M \rightarrow M^{* *}=F$ is injective and via this map, $M$ is canonically identified as a submodule of $F$. Then, since the functor ()$^{*}$ is compatible with localization on finitely generated $R$-modules and since for any height 1 prime $P$ of $R$, the finitely generated torsion-free module $M_{P}$ over the discrete valuation ring $R_{P}$ is free, it follows that $M_{P}=F_{P}$ for any such $P$. Therefore the quotient module $F / M$ is supported only at the maximal ideal $\mathbf{m}$ of $R$ and is of finite length.

Next, let $G$ be a free $R$-module containing $M$ with $G / M$ of finite length. The short exact sequence

$$
0 \longrightarrow M \longrightarrow G \longrightarrow G / M \longrightarrow 0
$$

on dualizing gives the exact sequence

$$
0 \longrightarrow G^{*} \longrightarrow M^{*} \longrightarrow \operatorname{Ext}_{R}^{1}(G / M, R) \longrightarrow 0 .
$$

The module $(G / M)^{*}$ vanishes since $G / M$ is of finite length and $\operatorname{Ext}_{R}^{1}(G, R)$ vanishes since $G$ is free.

Now, since $\operatorname{Ext}_{R}^{1}(G / M, R)$ is of finite length and the modules $G^{*}$ and $M^{*}$ are free, it follows from the Auslander-Buchsbaum formula again that $\operatorname{Ext}_{R}^{1}(G / M, R)=0$. Thus the map $G^{*} \longrightarrow M^{*}$ is an isomorphism and a further dualization gives an isomorphism $F \cong M^{* *} \longrightarrow G^{* *} \cong G$. By functoriality of the construction, this isomorphism restricts to the identity map on $M$.

To show uniqueness of such an isomorphism, consider the difference of two such. This is a map $F \stackrel{\phi}{\longrightarrow} G$ that vanishes on $M$. But since $F / M$ is of finite length, for some $t>0$, we have that $\mathbf{m}^{t} F \subseteq M \Rightarrow \phi\left(\mathbf{m}^{t} F\right)=0 \Rightarrow \mathbf{m}^{t} \phi(F)=0$. But $\phi(F)$ is a submodule of the free module $G$. So it follows that $\phi(F)=0 \Rightarrow$ $\phi=0$.

Let $\operatorname{rank}_{R}(M)$ denote the rank of $M$ and $\nu_{R}(M)$ denote the minimal number of generators of $M$. Recall that the rank of $M$ is the vector space dimension of the $K$-vector space $M_{K}=M \otimes_{R} K$. In our setting, $M \otimes_{R} K=F \otimes_{R} K$ since $F / M$ is of finite length and so $\operatorname{rank}_{R}(M)=\operatorname{rank}_{R}(F)$. But $F$ is free and so $\operatorname{rank}_{R}(F)=\nu_{R}(F)$.

Choose a basis of $F$ and a set of generators of $M$ and consider the matrix expressing this set of generators in terms of the chosen basis of $F$. We consider the elements of $F$ as being column vectors and so the resulting matrix is a $\operatorname{rank}_{R}(M) \times \nu_{R}(M)$ matrix. The ideal of maximal minors, i.e., $\operatorname{rank}_{R}(M)$ sized minors, of this matrix is easily seen to be independent of the choices made and is an invariant of $M$ which will be denoted $I(M)$. We define the order of $M$, denoted $\operatorname{ord}_{R}(M)$, to be $\operatorname{ord}_{R}(I(M))$.

The next proposition is an analog for modules of the result $\nu_{R}(I) \leq \operatorname{ord}_{R}(I)+$ 1 that holds for an m-primary ideal $I$. The proof for ideals in [Hnk] uses the Hilbert-Burch theorem. For modules, we use the Buchsbaum-Eisenbud structure theorem.

Proposition 2.2. Let $M$ be a finitely generated torsion-free $R$-module. Then, $\nu_{R}(M) \leq \operatorname{ord}_{R}(M)+\operatorname{rank}_{R}(M)$.

Proof. If $M$ is free, then $F=M$ and $I(M)=R$ so that $\operatorname{ord}_{R}(M)=0$. Also, 
$\nu_{R}(M)=\operatorname{rank}_{R}(M)$ and so equality holds in the proposition. Next, consider the case that $M$ is not free.

Consider a free resolution of $F / M$ of the form

$$
0 \longrightarrow K \stackrel{\psi}{\longrightarrow} G \stackrel{\phi}{\longrightarrow} F \longrightarrow 0
$$

where $G$ is chosen to map minimally onto $M$ so that $\operatorname{rank}_{R}(G)=\nu_{R}(M)$. Since $F / M$ is a non-zero $R$-module of finite length, $K \neq 0$. Let $I(\phi)$ and $I(\psi)$ denote the ideals of maximal minors of matrices representing $\phi$ and $\psi$ with respect to some (any) choice of bases for $F, G$ and $K$. Since $F / M$ is of finite length, the above sequence is split exact after localizing at any height 1 prime of $R$ and it follows from this that $I(\phi)$ and $I(\psi)$ are both m-primary ideals. Note that by definition, $I(M)=I(\phi)$. A corollary of the BuchsbaumEisenbud structure theorem ([BchSnb, Corollary 5.1]) asserts that $I(\phi) \cong I(\psi)$ as ideals of $R$. Since these are both $\mathbf{m}$-primary ideals, this implies that they are equal. Hence $\operatorname{ord}_{R}(M)=\operatorname{ord}_{R}(I(M))=\operatorname{ord}_{R}(I(\phi))=\operatorname{ord}_{R}(I(\psi))$.

Finally, as $G$ is chosen to map minimally onto $M$, any matrix representing $\psi$ has all its entries in $\mathbf{m}$ and so $\operatorname{ord}_{R}(I(\psi)) \geq \operatorname{rank}_{R}(K)=\operatorname{rank}_{R}(G)-$ $\operatorname{rank}_{R}(F)=\nu_{R}(M)-\operatorname{rank}_{R}(M)$. This completes the proof.

Observe that from the proof, it follows that $I(M)$ is always m-primary (or the whole of $R$ if $M$ is free).

We will refer to rings between $R$ and its field of fractions $K$ as birational overrings of $R$. For such a birational overring $S$, let $M S$ be the $S$-submodule of $M_{K}=M \otimes_{R} K$ generated by $M$. Note that $M S$ is contained in the free $S$-module $F S$. As an $S$-module, $M S$ is isomorphic to $M \otimes_{R} S$ modulo $S$ torsion. We call $M S$ the transform of $M$ in $S$. The module $M$ is said to be contracted from $S$ if $M=M S \cap F$ regarded as submodules of $F S$.

Note that for an ideal $I$ of $R$ its transform according to this definition is merely the extension to $S$ while in [Hnk] the transform is defined differently. However both definitions give isomorphic $S$-modules which is all that is required in this context.

The next two remarks will be needed in the proof of Proposition 2.5 which characterizes modules contracted from birational overrings of the form $S=$ $R\left[\frac{\mathbf{m}}{x}\right]$ for a minimal generator $x$ of $\mathbf{m}$. The function $\lambda_{R}(-)$ denotes the length function on $R$-modules.

Remark 2.3. $\nu_{R}(M)-\operatorname{rank}_{R}(M)=\lambda_{R}\left(\left(M:_{F} \mathbf{m}\right) / M\right)$.

Proof. Consider calculation of $\operatorname{Tor}_{2}^{R}(F / M, k)$ in two different ways. First, the resolution of $F / M$ in Proposition 2.2 can be used to calculate it. Since $\psi$ vanishes on tensoring with $k$, we have $\operatorname{dim}_{k}\left(\operatorname{Tor}_{2}^{R}(F / M, k)\right)=\operatorname{rank}_{R}(K)$. But $\operatorname{rank}_{R}(K)=\operatorname{rank}_{R}(G)-\operatorname{rank}_{R}(F)=\nu_{R}(M)-\operatorname{rank}_{R}(M)$.

Secondly, the Koszul resolution

$$
0 \longrightarrow R \longrightarrow R^{2} \longrightarrow R \longrightarrow 0
$$

of $k$ can be used to calculate it and shows that $\operatorname{Tor}_{2}^{R}(F / M, k) \cong\left(M:_{F} \mathbf{m}\right) / M$. Comparing these calculations proves the remark.

Remark 2.4. For a minimal generator $x \in \mathbf{m}$,

$$
\lambda_{R}(F /(x F+M))=\lambda_{R}(R /(x, I(M))) .
$$


Proof. Let - denote going modulo $x$ and consider the free module $\bar{F}$ over the discrete valuation ring $\bar{R}$. The image of $M$ in $\bar{F}$ is a free submodule and of maximal rank since $F / M$ and hence also $F /(x F+M)$ are of finite length. By the structure theorem for modules over a principal ideal domain, in an appropriate basis of $\bar{F}$, the image of $M$ is generated by a set of generators represented by a diagonal matrix. Then the image of $I(M)$ in $\bar{R}$ is generated by the product of the diagonal elements. The remark follows from these observations.

Proposition 2.5. With $M$ a finitely generated torsion-free $R$-module and $F=$ $M^{* *}$, the following conditions are equivalent:

(1) There exists $x \in \mathbf{m}-\mathbf{m}^{2}$ so that $M$ is contracted from $S=R\left[\frac{\mathbf{m}}{x}\right]$.

(2) There exists $x \in \mathbf{m}-\mathbf{m}^{2}$ so that $\left(M:_{F} x\right)=\left(M:_{F} \mathbf{m}\right)$.

(3) There exists $x \in \mathbf{m}-\mathbf{m}^{2}$ so that $\lambda_{R}(F /(x F+M))=\nu_{R}(M)-\operatorname{rank}_{R}(M)$.

(4) $\operatorname{ord}_{R}(M)=\nu_{R}(M)-\operatorname{rank}_{R}(M)$.

(5) For any $x \in \mathbf{m}$ such that $\operatorname{ord}_{R}(I(M))=\lambda_{R}(R /(x, I(M))), M$ is contracted from $S=R\left[\frac{\mathrm{m}}{x}\right]$.

Proof. (1) $\Rightarrow(2)$. It suffices to show that for $v \in F$, if $x v \in M$ then $\mathbf{m} v \subseteq M$. But $\mathbf{m} v=x v \cdot \frac{\mathbf{m}}{x} \subseteq M S \cap F=M$.

$(2) \Leftrightarrow(3)$. The exact sequence of finite length $R$-modules

$$
0 \longrightarrow \frac{\left(M:_{F} x\right)}{M} \longrightarrow \frac{F}{M} \stackrel{x}{\longrightarrow} \frac{F}{M} \longrightarrow \frac{F}{(x F+M)} \longrightarrow 0
$$

and additivity of length show that $\lambda_{R}\left(\left(M:_{F} x\right) / M\right)=\lambda_{R}(F /(x F+M))$. Using this together with Remark 2.3 gives that $\left(M:_{F} x\right) / M=\left(M:_{F} \mathbf{m}\right) / M$ if and only if $\lambda_{R}((F / x F+M))=\nu_{R}(M)-\operatorname{rank}_{R}(M)$.

(3) $\Rightarrow$ (4). By Remark 2.4, (3) $\Rightarrow \lambda_{R}(R /(x, I(M)))=\nu_{R}(M)-\operatorname{rank}_{R}(M)$. Hence $\operatorname{ord}_{R}(M)=\operatorname{ord}_{R}(I(M)) \leq \lambda_{R}(R /(x, I(M)))=\nu_{R}(M)-\operatorname{rank}_{R}(M)$. As the reverse inequality always holds by Proposition 2.2 , we obtain equality.

$(4) \Rightarrow(5)$. Let $x \in \mathbf{m}$ be such that $\operatorname{ord}_{R}(I(M))=\lambda_{R}(R /(x, I(M)))$. By Remark 2.4 and (4) we have that for such $x, \nu_{R}(M)-\operatorname{rank}_{R}(M)=\lambda_{R}(F /(x F+$ $M))$. By the equivalence of (2) and (3), then, $\left(M:_{F} x\right)=\left(M:_{F} \mathbf{m}\right)$. Consider $S=R\left[\frac{\mathrm{m}}{x}\right]=\bigcup_{n>0} \frac{\mathbf{m}^{n}}{x^{n}}$. Then $(M S \cap F)=\bigcup_{n \geq 0}\left(\mathbf{m}^{n} M: F x^{n}\right)$. To show that this is equal to $\bar{M}$ we induce on $n$ to show that each term of the union is $M$. The basis case $n=0$ is clear. For $n>0$ and $v \in F$, suppose that $x^{n} v \in$ $\mathbf{m}^{n} M=y^{n} M+x \mathbf{m}^{n-1} M$ where $\mathbf{m}=(x, y)$. Then write $x^{n} v=y^{n} v^{\prime}+x a v^{\prime \prime}$ where $a \in \mathbf{m}^{n-1}$ and $v^{\prime}, v^{\prime \prime} \in M$. So $x\left(x^{n-1} v-a v^{\prime \prime}\right)=y^{n} v^{\prime}$. Reading this equation in $F$, we get that $v^{\prime}=x w$ and $x^{n-1} v-a v^{\prime \prime}=y^{n} w$ for some $w \in F$. Finally, since $x w=v^{\prime} \in M, w \in\left(M:_{F} x\right)=\left(M:_{F} \mathbf{m}\right)$ and so $y^{n} w \in M \mathbf{m}^{n-1} \Rightarrow v \in\left(M \mathbf{m}^{n-1}:_{F} x^{n-1}\right)=M$.

$(5) \Rightarrow(1)$. That such an $x$ exists is clear. For instance, if $a \in I(M)$ is an element of minimal order, then any $x$ whose leading form in the associated graded ring $\operatorname{gr}_{\mathrm{m}}(R)$ does not divide the leading form of $a$ works.

Proposition 2.6. Let $B \subseteq F$ be finitely generated free $R$-modules of equal rank. For some choice of bases of $F$ and $B$, let $\widetilde{B}$ be the square matrix whose columns are the coordinate representations of the basis of $B$ in terms of that of $F$. For a prime element $x$ of $R$, the following three conditions are equivalent:

(1) $x$ does not divide $\operatorname{det}(\widetilde{B})$ in $R$. 
(2) $\left(B:_{F} x\right)=B$.

(3) $\operatorname{Tor}_{1}^{R}(F / B, R / x R)=0$.

Proof. (1) $\Rightarrow(2)$. Suppose that $v \in\left(B:_{F} x\right)$. Then $x v \in B$. Let the column vector $\widetilde{v}$ represent $v$ in terms of the basis of $F$. Since $x v \in B, x \widetilde{v}=\widetilde{B} \tilde{w}$ for some column vector $\widetilde{w}$. Multiplying both sides by the adjoint matrix $\operatorname{adj}(\widetilde{B})$ gives $x \operatorname{adj}(\widetilde{B}) \widetilde{v}=\operatorname{det}(\widetilde{B}) \widetilde{w}$. Since $x$ is a prime element of $R$ and does not divide $\operatorname{det}(\widetilde{B})$, it follows that $x$ divides every entry of the column matrix $\widetilde{w}$. Hence $\widetilde{w}=x \widetilde{u}$ for some matrix $\tilde{u}$. But then, $x \widetilde{v}=\widetilde{B} \widetilde{w} \Rightarrow x \widetilde{v}=x \widetilde{B} \widetilde{u} \Rightarrow \widetilde{v}=$ $\widetilde{B} \tilde{u}$. Therefore $v \in B$.

(2) $\Leftrightarrow$ (3). Calculating $\operatorname{Tor}_{1}^{R}(F / B, R / x R)$ from the resolution

$$
0 \rightarrow R \stackrel{x}{\longrightarrow} R \rightarrow 0
$$

shows that it is isomorphic to $\left(B:_{F} x\right) / B$. The equivalence follows.

(3) $\Rightarrow$ (1). Vanishing of $\operatorname{Tor}_{1}^{R}(F / B, R / x R)$ implies that (with - denoting modulo $x$ )

$$
0 \rightarrow \bar{F} \stackrel{\widetilde{B}}{\longrightarrow} \bar{F}
$$

is exact. Hence $\operatorname{det}(\widetilde{B})$ is not 0 in $\bar{R}$. Lift back to $R$.

Suppose that $M$ and $N$ are finitely generated torsion-free $R$-modules. We define their product, denoted $M N$, to be $M \otimes_{R} N$ modulo $R$-torsion. Equivalently, if $F$ and $G$ are free $R$-modules containing $M$ and $N$ respectively, then $M N$ is the image in $F \otimes_{R} G$ of $M \otimes_{R} N$. Hence, if after a choice of bases of $F$ and $G$, the matrices $\widetilde{M}$ and $\widetilde{N}$ have columns generating $M$ and $N$, then the Kronecker product or the outer product $\widetilde{M} \otimes \widetilde{N}$ of the matrices has columns generating $M N$ for a suitable basis of $F \otimes_{R} G$. Recall that the outer product of two matrices is obtained by replacing each entry of one of them by the product of that entry with the other matrix.

For a finitely generated torsion-free $R$-module $M$, and an integer $n \geq 0$, set $S_{n}(M)=\operatorname{Sym}_{n}(M)$ modulo $R$-torsion where $\operatorname{Sym}_{n}(M)$ denotes the $n^{\text {th }}$ symmetric power of $M$. We will refer to $S_{n}(M)$ as the torsion-free $n^{\text {th }}$ symmetric power of $M$. For $M$ contained in a free $R$-module $F$, we have that $S_{n}(M)$ is the image of $\operatorname{Sym}_{n}(M)$ in $\operatorname{Sym}_{n}(F)$. In terms of matrices, if $M$ is generated by the columns of $\widetilde{M}$, then, $S_{n}(M)$ is generated by the columns of the matrix $S_{n}(\widetilde{M})$. These columns are obtained by regarding the columns of $\widetilde{M}$ as linear forms in a basis of $F$ and then taking the coefficients of the monomials of degree $n$ in these forms.

Finally, for a finitely generated torsion-free $R$-module $M$ and an integer $n \geq 0$, set $E_{n}(M)=\bigwedge_{n}(M)$ modulo $R$-torsion where $\bigwedge_{n}(M)$ denotes the $n^{\text {th }}$ exterior power of $M$. The $E_{n}(M)$ will be called the torsion-free $n^{\text {th }}$-exterior power of $M$. Again, if $F$ is free and contains $M$, then $E_{n}(M)$ is the image of $\bigwedge_{n}(M)$ in $\bigwedge_{n}(F)$. In particular $E_{n}(M)$ vanishes for $n>\operatorname{rank}_{R}(M)$. In matrix terms, a column of $E_{n}(M)$ is obtained by choosing $n$ columns of $\widetilde{M}$ and taking the $n$-sized minors of the submatrix obtained.

Corollary 2.7. Let $B \subseteq F$ and $C \subseteq G$ be pairs of finitely generated free $R$ modules such that $B$ and $F$ have equal rank and $C$ and $G$ have equal rank 
and let $\widetilde{B}$ and $\widetilde{C}$ be matrices whose columns are minimal generators of $B$ and $C$ with respect to some chosen bases of $F$ and $G$. Suppose that $x$ is a prime element of $R$.

(1) $\left(B C: F \otimes_{R} G x\right)=B C$ if and only if $x$ does not divide each of $\operatorname{det}(\widetilde{B})$ and $\operatorname{det}(\widetilde{C})$.

(2) $\left(S_{n}(B): \operatorname{Sym}_{n}(F) x\right)=S_{n}(B)$ if and only if $x$ does not divide $\operatorname{det}(\widetilde{B})$.

(3) $\left(E_{n}(B): \bigwedge_{n}(F) x\right)=E_{n}(B)$ if and only if $x$ does not divide $\operatorname{det}(\widetilde{B})$.

Proof. (1). By the above remarks, in an appropriate basis of $F \otimes_{R} G$, a matrix of generators of $B C$ is the outer product of the square matrices $\widetilde{B}$ and $\widetilde{C}$. Since $\operatorname{det}(\widetilde{B} \otimes \widetilde{C})=\operatorname{det}(\widetilde{B})^{\operatorname{size}(C)} \cdot \operatorname{det}(\widetilde{C})^{\operatorname{size}(B)}$, the outer product of the non-singular square matrices $\widetilde{B}$ and $\widetilde{C}$ is still non-singular and so $B C$ is a free submodule of $F \otimes_{R} G$ with maximal rank. In fact, it follows that the prime element $x$ does not divide $\operatorname{det}(\widetilde{B} \otimes \widetilde{C})$. Now, Proposition 2.6 shows that $\left(B C: F \otimes_{R} G\right)=B C$.

The proofs of (2) and (3) are similar and essentially follow from the observations that $\operatorname{det}\left(S_{n}(\widetilde{B})\right)$ and $\operatorname{det}\left(E_{n}(\widetilde{B})\right)$ are each a power of $\operatorname{det}(\widetilde{B})$ with appropriate binomial coefficient exponents.

We are now prepared to prove the main result of this section that asserts that products, torsion-free symmetric powers and torsion-free exterior powers of contracted modules are contracted. The utility of this result is that once integrally closed modules are shown to be contracted, this theorem provides a way to prove that they are closed under products, torsion-free symmetric powers and torsion-free exterior powers by going to a larger ring and then contracting back.

Theorem 2.8. Let $M$ and $N$ be finitely generated torsion-free $R$-modules that are both contracted from $S=R\left[\frac{\mathbf{m}}{x}\right]$ for some $x \in \mathbf{m}-\mathbf{m}^{2}$. Then

(1) Their product $M N$ is also contracted from $S$.

(2) For every $n \geq 0$, the module $S_{n}(M)$ is contracted from $S$.

(3) For every $n \geq 0$, the module $E_{n}(M)$ is contracted from $S$.

Proof. Let $F$ and $G$ be the double duals of $M$ and $N$ respectively. So we canonically have $M \subseteq F$ and $N \subseteq G$ with finite length quotients. Let denote going modulo $x$ and consider the image of $M$ in the free $\bar{R}$-module $\bar{F}$. This is also a free $\bar{R}$-module of rank equal to $\operatorname{rank}_{\bar{R}}(\bar{F})=\operatorname{rank}_{R}(M)$. Choose $\operatorname{rank}_{R}(M)$ elements in $M$ that minimally generate its image in $\bar{F}$. Let $B$ be the submodule of $M$ generated by these. $B$ is a free submodule of $F$ of maximal rank since elements of $F$ that are linearly independent modulo $x$ are already linearly independent. Note that as the image of $B$ in $\bar{F}$ is free of maximal rank, $x$ does not divide $\operatorname{det}(\widetilde{B})$. We have that $M=B+(x F \cap M)=B+x\left(M:_{F} x\right)$. Similarly choose a $C \subseteq N$ such that $N=C+x\left(N:_{G} x\right)$ with $x$ not dividing $\operatorname{det}(\widetilde{C})$.

(1) Let $H$ denote $F \otimes_{R} G$ and consider $M N=\operatorname{im}\left(\left(M \otimes_{R} N\right) \longrightarrow H\right)$. By right-exactness of the tensor product, $M N$ is of finite colength in $H$. Therefore $H$, by Proposition 2.1 , can be regarded canonically as the double dual of $M N$. The proof of Proposition 2.5 shows that to see that $M N$ is contracted from $S$, it is enough to see that $\left(M N:_{H} x\right)=\left(M N:_{H} \mathbf{m}\right)$. 
From the definitions, $M N=B C+x M\left(N:_{G} x\right)+x N\left(M:_{F} x\right)$, where the equation holds as submodules of $H$. To show that $\left(M N:_{H} x\right)=\left(M N:_{H} \mathbf{m}\right)$ suppose $v \in\left(\begin{array}{lll}M N & :_{H} & x\end{array}\right)$. Then $v \in H$ and $x v \in M N=B C+$ $x M\left(N:_{G} x\right)+x N\left(M:_{F} x\right)$. So for some $v^{\prime} \in M\left(N:_{G} x\right)$ and $v^{\prime \prime} \in N\left(M:_{F} x\right)$ we have $x\left(v-v^{\prime}-v^{\prime \prime}\right) \in B C$. But by Corollary 2.7, $\left(B C:_{F} x\right)=B C$. Hence $v \in B C+M\left(N:_{G} x\right)+N\left(M:_{F} x\right)$. Therefore $\mathbf{m} v \subseteq \mathbf{m} B C+M \mathbf{m}\left(N:_{G} x\right)$ $+\mathbf{m}(M: F \quad x) N \subseteq M N$ since $M$ and $N$ are contracted from $S$ and so $\mathbf{m}\left(M:_{F} x\right) \subseteq M$ and $\mathbf{m}\left(N:_{F} x\right) \subseteq N$.

(2) Let $H$ denote $\operatorname{Sym}_{n}(F)$ for some fixed $n \geq 1 . S_{n}(M)$ is the image in $H$ of $\operatorname{Sym}_{n}(M)$. As before, $S_{n}(M)$ is of finite colength in $H$ and we need to see that $\left(S_{n}(M):_{H} x\right)=\left(S_{n}(M):_{H} \mathbf{m}\right)$.

For submodules $Q_{1}, Q_{2}, \cdots, Q_{n}$ of $F$, define $Q_{1} * Q_{2} * \cdots * Q_{n}$ to be the image of $Q_{1} \otimes Q_{2} \otimes \cdots \otimes Q_{n}$ in $H$. Thus $S_{n}(M)=M * M * \cdots * M$ ( $n \quad M$ 's). Since $M=B+x\left(M:_{F} x\right)$, it follows that $S_{n}(M)=S_{n}(B)+$ $x\left(M:_{F} x\right) * M * M * \cdots * M\left(n-1 \quad M\right.$ 's). Now suppose that $v \in\left(S_{n}(M):_{H}\right.$ $x)$. Then $x v \in S_{n}(B)+x\left(M:_{F} x\right) * M * M * \cdots * M$. So for some $v^{\prime} \in$ $\left(M:_{F} x\right) * M * M * \cdots * M$, we have that $x\left(v-v^{\prime}\right) \in S_{n}(B)$. By Corollary 2.7, $v-v^{\prime} \in S_{n}(B)$. Hence $\mathbf{m} v \in \mathbf{m} S_{n}(B)+\mathbf{m}\left(M:_{F} x\right) * M * M * \cdots * M \subseteq S_{n}(M)$ since $\left(M:_{F} x\right)=\left(M:_{F} \mathbf{m}\right)$.

(3) If now $H$ denotes $\Lambda_{n}(F)$ for some fixed $n \geq 1$ and for submodules $Q_{1}$, $Q_{2}, \cdots, Q_{n}$ of $F$, we let $Q_{1} * Q_{2} * \cdots * Q_{n}$ denote the image of $Q_{1} \otimes Q_{2} \otimes \cdots \otimes Q_{n}$ in $H$, the proof of (2) goes through to show that $E_{n}(M)$ is contracted from $S$.

\section{INTEGRAL CLOSURE AND BUCHSBAUM-RIM MULTIPLICITY OF MODULES}

In this section we review the notions of reductions and integral closures of modules as developed by Rees in [Rs] and the notion of the Buchsbaum-Rim multiplicity of a module as developed by Buchsbaum and Rim in [BchRm]. The term Buchsbaum-Rim multiplicity is due to Kirby in [Krb].

The theory of reductions and integral closures of modules can be developed for torsion-free modules over an arbitrary Noetherian domain without assumptions of locality or regularity. So we let $R$ be a Noetherian domain with field of fractions $K$ and let $M$ be a finitely generated torsion-free $R$-module. By $M_{K}$ we denote the finite-dimensional $K$-vector space $M \otimes_{R} K$. If $N$ is a submodule of $M$, then $N_{K}$ is naturally identified with a subspace of $M_{K}$.

For any birational overring $S$ of $R$, we use the notation $M S$ to be the $S$-submodule of $M_{K}$ generated by $M$. In the special case when $R$ is a twodimensional regular local ring, recall that $M S$ is the so-called transform of $M$ in $S$. In this section, the birational overrings of $R$ that we will focus on are the discrete valuation rings of $K$ containing $R$.

Definition 3.1. With notation as above, an element $u \in M_{K}$ is said to be integral over $M$ if $u \in M V$ for every discrete valuation ring $V$ of $K$ containing $R$. The integral closure of $M$, denoted $\bar{M}$, is the set of all elements of $M_{K}$ that are integral over $M$. The module $M$ is said to be integrally closed if $M=\bar{M}$. A submodule $N$ of $M$ is said to be a reduction of $M$ if $M \subseteq \bar{N}$.

We begin with some immediate consequences of this definition. First, it is clear that $\bar{M}$ is an $R$-submodule of $M_{K}$ which contains $M$. In fact, since 
any valuation ring of $K$ which contains $R$ also contains its integral closure $\bar{R}$ in $K$, we have that $\bar{M}$ is an $\bar{R}$-module. Rees shows in [Rs] that $\bar{M}$ is a finitely generated $R$-module exactly when $\bar{R}$ is a finitely generated $R$-module. He also shows that in this case, if $\left({ }_{-}\right)^{\dagger}$ denotes the functor $\operatorname{Hom}_{R}(-, \bar{R})$, then $M^{\dagger \dagger}$ can be naturally identified with a submodule of $M_{K}$ and that $\bar{M} \subseteq M^{\dagger \dagger}$. Thus, if $R$ is a Noetherian normal domain, and so in particular if it is a twodimensional regular local ring, this implies that $\bar{M} \subseteq M^{* *}$, where $\left({ }^{-}\right)^{*}$ as usual denotes $\operatorname{Hom}_{R}(-, R)$.

Further, if $M$ and $N$ are finitely generated torsion-free $R$-modules that are integrally closed, then so is their direct sum $M \oplus N$. Over a Noetherian normal domain, each integrally closed ideal is an integrally closed module in the sense of the above definition. Thus a good source of examples of integrally closed modules of large rank over such a ring is obtained simply by taking direct sums of integrally closed ideals. In one sense, these are trivial as they are decomposable. In the last section we give an explicit construction of integrally closed indecomposable modules.

Before we state the next result, we will require some notations and results about symmetric and exterior powers and algebras associated to a module.

For a Noetherian domain $R$ and a finitely generated torsion-free $R$-module $M$, by $S(M)$ we will denote the image of the symmetric algebra $\operatorname{Sym}^{R}(M)$ in $\operatorname{Sym}^{K}\left(M_{K}\right)$ under the canonical map. As an $R$-algebra, $S(M)=\operatorname{Sym}^{R}(M)$ modulo its ideal of $R$-torsion elements. The symmetric algebra $\operatorname{Sym}^{R}(M)$ is a graded algebra whose graded components are the symmetric powers of $M$ and similarly $S(M)$ is a graded algebra whose graded components are the torsionfree symmetric powers of $M$. One fact that we will need is that the integral closure of one graded domain in another is still naturally graded. Note that since $\operatorname{Sym}^{K}\left(M_{K}\right)$ is a polynomial ring over $K$ and hence integrally closed, $\overline{S(M)} \subseteq \operatorname{Sym}^{K}\left(M_{K}\right)$ and is thus a graded domain. In particular, the homogeneous component of degree 1 of $\overline{S(M)}$ can be identified with a submodule of $\operatorname{Sym}_{1}^{K}\left(M_{K}\right)=M_{K}$.

We will not deal with the exterior algebra of $M$ as an object but only with its graded components $\Lambda_{n}(M)$ and the torsion-free exterior powers $E_{n}(M)$. Note that $E_{n}(M)$ can be regarded as the image of $\Lambda_{n}(M)$ in $\Lambda_{n}\left(M_{K}\right)$-the latter exterior power over the field $K$. Thus, when $n=\operatorname{rank}_{R}(M), E_{n}(M)$ is an $R$-submodule of $K$ and therefore isomorphic to an ideal of $R$. Also note that in this case if $N \subseteq M \subseteq N_{K}$ then, $E_{n}(N) \subseteq E_{n}(M)$ and fixing an isomorphism of $E_{n}(M)$ with an ideal of $R$ identifies $E_{n}(N)$ as a subideal.

We are now prepared to state a fundamental theorem due to Rees ([Rs, Theorems $1.2,1.5]$ ) which gives three different characterizations of when an element is integral over a module.

Theorem 3.2. Let $R$ be a Noetherian domain and let $M$ be a finitely generated torsion-free $R$-module of rank $r$. For an element $v \in M_{K}$, the following conditions are equivalent:

(1) The element $v$ is integral over $M$.

(2) The element $v \in M_{K}=\operatorname{Sym}_{1}^{K}\left(M_{K}\right)$ is integral over $S(M)$.

(3) Under some isomorphism of $E_{r}(M+R v)$ with an ideal $I$ of $R$, the subideal $J$ corresponding to $E_{r}(M)$ is a reduction of $I$. 
The three criteria of the theorem, in order, will be referred to as the valuative, equational and determinantal criteria for integral dependence. The following corollary of Theorem 3.2 is a local-global principle for integral dependence on a module.

Corollary 3.3. Let $R$ be Noetherian normal domain and $M$ be a finitely generated torsion-free $R$-module. The $R$-module $M$ is integrally closed if and only if for every maximal ideal $\mathbf{m}$ of $R$, the $R_{\mathbf{m}}$-module $M_{\mathbf{m}}$ is integrally closed.

Proof. First, suppose that each $M_{\mathrm{m}}$ is an integrally closed $R_{\mathrm{m}}$-module. Consider an element $v \in M_{K}$ that is integral over $M$. By definition, it is in $M V$ for every discrete valuation ring $V$ of $K$ that contains $R$. Hence, for every maximal ideal $\mathbf{m}$ of $R$, and every discrete valuation ring of $K$ containing $R_{\mathrm{m}}, v$ is in $M_{\mathrm{m}} V=M V$. Since each $M_{\mathrm{m}}$ is integrally closed, it follows that $v \in M_{\mathbf{m}}$ for every maximal ideal $\mathbf{m}$ of $R$. Therefore $v \in M$.

Next, suppose that $M$ is an integrally closed $R$-module and that $\mathbf{m}$ is a maximal ideal of $R$. To show that $M_{\mathrm{m}}$ is integrally closed, consider an element $v \in M_{K}$ integral over $M_{\mathrm{m}}$. By the equational criterion, $v$ satisfies an integral equation over the image $S\left(M_{\mathbf{m}}\right)$ of $\operatorname{Sym}^{R_{\mathrm{m}}}\left(M_{\mathrm{m}}\right)$ in $\operatorname{Sym}^{K}\left(M_{K}\right)$. Any element in $S\left(M_{\mathrm{m}}\right)$ is multiplied into $S(M)$ by some element of $R$ not in $\mathbf{m}$. Suppose that $s \in R-\mathbf{m}$ multiplies every coefficient of the equation for $v$ into $S(M)$. Then $s v$ is integral over $M$ and, since $M$ is integrally closed, is in $M$. So $v \in M_{\mathbf{m}}$.

Another result of Rees ([Rs, Lemma 2.2]) generalizes to modules the well known theorem that any m-primary ideal of a $d$-dimensional Noetherian local ring $(R, \mathbf{m})$ with infinite residue field has a $d$-generated reduction.

Theorem 3.4. Let $R$ be a d-dimensional Noetherian local domain with infinite residue field and $M$ be a finitely generated torsion-free $R$-module. Then $M$ has a reduction which is generated by $\operatorname{rank}_{R}(M)+d-1$ elements.

It is also true that in the situation of the above theorem, if $M$ is contained in a free $R$-module $F$ with finite length quotient, then no reduction of $M$ is generated by fewer then $\operatorname{rank}_{R}(M)+d-1$ elements. Below, we discuss in detail an example to illustrate these theorems and some of the results of the previous section.

Example 3.5. Let $R=k[[x, y]]$ be a ring of formal power series over a field $k$ and let $K$ denote its field of fractions. Denote by $F$ the free $R$-module $R \oplus R$ of rank 2 whose elements will be represented by column matrices with two entries. Let $M$ be the submodule of $F$ generated by the columns of the matrix

$$
\widetilde{M}=\left(\begin{array}{lll}
x & y & 0 \\
0 & x & y
\end{array}\right) .
$$

As a matter of notation, let $T$ be the column vector $\left[\begin{array}{ll}1 & 0\end{array}\right]^{*}$ and $U$ be the column vector $\left[\begin{array}{ll}0 & 1\end{array}\right]^{*}$ (with $*$ denoting transpose). $M$ is then the submodule of $F$ generated by $x T, y T+x U$ and $y U$. Since $x(y T+x U)-y(x T)=x^{2} U$ and $y(y T+x U)-x(y U)=y^{2} T$, the module $M$ contains the direct sum of ideals $\left(x, y^{2}\right) \oplus\left(y, x^{2}\right)$. It follows that $\lambda_{R}(F / M)$ is finite and hence, by Proposition 2.1, $F$ can be canonically identified with the double dual of $M$. 
Consider the element $y T$ of $F$. We will show that this element is integral over $M$ using each of the criteria of Theorem 3.2. First, let $V$ be any discrete valuation ring of $K$ containing $R$. To show that $y T$ is integral over $M$, it must be seen that $y T \in M V$. Consider the ideal $(x, y) V$ of the local ring $V$. Since it is generated by $x$ and $y$ and is principal, one of $x$ or $y$ generate it. Say $x V=(x, y) V$ so that $y / x \in V$. Then, $y T=(y / x)(x T) \in M V$. Similarly, if $y V=(x, y) V$ then $y T=(y T+x U)-(x / y)(y U) \in M V$. So by the valuative criterion, $y T \in \bar{M}$.

Next, consider $S(M) \subseteq S(F) \subseteq \operatorname{Sym}^{K}\left(M_{K}\right)=\operatorname{Sym}^{K}\left(F_{K}\right)$. By definition, $S(F)$ is the image of $\operatorname{Sym}^{R}(F)$ in $\operatorname{Sym}^{K}\left(M_{K}\right)$. We may identify $\operatorname{Sym}^{K}\left(M_{K}\right)$ with a polynomial ring in two variables $T$ and $U$ over $K$ and then $S(F)$ is its subring $R[T, U]$. Furthermore, $S(M)$ is the subring $R[x T, y T+x U, y U]$ of $R[T, U]$. The element $y T$ of $R[T, U]$ satisfies the integral equation $(y T)^{2}-$ $(y T+x U) y T+(x T)(y U)=0$ over $R[x T, y T+x U, y U]$. Therefore, by the equational criterion, $y T$ is integral over $M$.

Lastly, consider the submodule $N$ of $F$ generated by $M$ and the element $y T$, i.e., the submodule of $F$ generated by the columns of the matrix

$$
\tilde{N}=\left(\begin{array}{llll}
x & y & 0 & y \\
0 & x & y & 0
\end{array}\right) \text {. }
$$

We have that $E_{2}(M) \subseteq E_{2}(N) \subseteq E_{2}(F)$. Since $F$ is a free $R$-module of rank $2, E_{2}(F)$ is isomorphic to $R$ and under any such isomorphism, $E_{2}(N)$ is identified with the ideal $\left(x^{2}, x y, y^{2}\right)$ of the $2 \times 2$ minors of $\widetilde{N}$. Also, $E_{2}(M)$ is identified with the ideal of $2 \times 2$ minors of $\widetilde{M}$ which also happens to be $\left(x^{2}, x y, y^{2}\right)$. Since any ideal is a reduction of itself, it follows from the determinantal criterion that $y T \in \bar{M}$.

We next show that $N$ is the integral closure of $M$. It only needs to be seen that $N$ is integrally closed. But from the matrix $\tilde{N}$, it is clear that $N \cong \mathbf{m} \oplus \mathbf{m}$ and is therefore integrally closed, being the direct sum of integrally closed ideals.

Note that $\nu_{R}(M)=3$ and $\nu_{R}(N)=4$ and that $I(M)=I(N)=\left(x^{2}, x y, y^{2}\right)$ so that $\operatorname{ord}_{R}(M)=\operatorname{ord}_{R}(N)=2$. So we have that $\nu_{R}(N)=\operatorname{ord}_{R}(N)+$ $\operatorname{rank}_{R}(N)$. This agrees with Proposition 2.5 in that $N$ is contracted from $S=R\left[\frac{\mathbf{m}}{x}\right]$ for some ( actually all ) $x \in \mathbf{m}-\mathbf{m}^{2}$. We will see in the next section that this is characteristic of all integrally closed modules over a two-dimensional regular local ring. Given this, that $M$ is not integrally closed follows simply from the numerical inequation $\nu_{R}(M) \neq \operatorname{ord}_{R}(M)+\operatorname{rank}_{R}(M)$.

We will next review the basic facts about the Buchsbaum-Rim multiplicity of modules. This concept of multiplicity was intoduced in 1964 by Buchsbaum and Rim as the alternating sum of the homology of a certain complex which is now called the Buchsbaum-Rim complex. Beginning with the papers of Kirby ([Krb]) and Kirby and Rees ([KrbRs]), there has been a revival of interest in this topic. A recent comprehensive paper of Kleiman and Thorup ([KlmThr]) provides a detailed introduction to the geometric theory of Buchsbaum-Rim multiplicity.

In the rest of this section, the notation will be as follows. $R$ will be a Noetherian local ring of dimension $d$, not neccesarily a domain. Let $P$ be an $R$-module of finite length with a free presentation

$$
G \longrightarrow F \longrightarrow P \longrightarrow 0 \text {. }
$$


Let $S(G)$ be the image of $\operatorname{Sym}^{R}(G)$ in $\operatorname{Sym}^{R}(F)=S(F)$. Then $S(G)$ is a graded subring of $S(F)$ whose homogeneous components are denoted $S_{n}(G)$. We begin by summarizing some results ([BchRm, Theorems 3.1-3.4]). A clear, recent exposition of parts of this theorem and several related results appears in [Ktz].

Theorem 3.6. If $P \neq 0$, then the length $\lambda_{R}\left(\operatorname{Sym}_{n}^{R}(F) / S_{n}(G)\right)$ is asymptotically given by a polynomial function of $n$ of degree $\operatorname{rank}_{R}(F)+d-1$. The normalized leading coefficient of this polynomial is independent of the presentation chosen.

Recall that the normalized leading coefficient of a numerical polynomial of degree $d$ is $d$ ! times its leading coefficient. The normalized leading coefficient above is an invariant of $P$ and is called the Buchsbaum-Rim multiplicity of $P$. We denote it by $e(P)$. We define the Buchsbaum-Rim multiplicity of the zero module to be 0 .

We require a property of the Buchsbaum-Rim multiplicity given by the following proposition ([BchRm, Corollary 4.5$])$. This property is analogous to the well known characterization of Cohen-Macaulay local rings as those for which the multiplicity of an ideal generated by a system of parameters is equal to its colength.

Proposition 3.7. Let $R$ be a Cohen-Maculay local ring of positive dimension $d$ and $F$ a finitely generated free $R$-module. Let $M \subseteq F$ be a submodule such that $\lambda_{R}(F / M)$ is finite. If $M$ is generated by $\operatorname{rank}_{R}(F)+d-1$ elements, then $e(F / M)=\lambda_{R}(F / M)$.

It follows fairly easily from the definition of reductions of ideals that an m-primary ideal and any reduction have the same multiplicity. In the case of modules, the proof is similar and we show this next for completeness.

Proposition 3.8. Let $R$ be a Noetherian local domain of positive dimension $d$ and $F$ be a finitely generated free $R$-module. Let $N \subseteq M$ be submodules of $F$ such that $\lambda_{R}(F / N)$ is finite. If $N$ is a reduction of $M$ then $e(F / N)=e(F / M)$. Proof. Consider free presentations of $F / M$ and $F / N$ of the form

$$
\begin{aligned}
& G \longrightarrow F \longrightarrow F / M \longrightarrow 0, \\
& H \longrightarrow F \longrightarrow F / N \longrightarrow 0 .
\end{aligned}
$$

By definition, $e(F / M)$ and $e(F / N)$ are the leading coefficients of the $\operatorname{rank}_{R}(F)$ $+d-1$ degree polynomials that give $\lambda_{R}\left(S_{n}(F) / S_{n}(G)\right)$ and $\lambda_{R}\left(S_{n}(F) / S_{n}(H)\right)$ for large $n$. Hence, to show that $e(F / M)=e(F / N)$, it is enough to show that the polynomial that gives $\lambda_{R}\left(S_{n}(M) / S_{n}(N)\right)$ for large $n$ is of strictly smaller degree then $\operatorname{rank}_{R}(F)+d-1$.

Consider the finitely generated graded $R$-algebras $S(N) \subseteq S(M)$. By the equational criterion, it follows that $S(M)$ is a finite $S(N)$ module since each of its generators is integral over $S(N)$. Choose a finite set of homogeneous generators for $S(M) / S(N)$ as an $S(N)$-module. Since each graded component of $S(M) / S(N)$ is of finite length, some power of $\mathbf{m}$ annihilates each of these generators and hence some power of $\mathrm{m} S(N)$ annihilates $S(M) / S(N)$.

Therefore, $S(M) / S(N)$ is a finitely generated graded module over the homogeneous graded ring $S(N) / \mathbf{m}^{k} S(N)$ for some $k>0$. To conclude the 
proof, it suffices to see that the dimension of the ring $S(N) / \mathbf{m}^{k} S(N)$ is at most $\operatorname{rank}_{R}(F)+d-1$, for then the length of the graded components of any finitely generated graded module over this ring will be given by a polynomial of strictly smaller degree.

The dimension of the ring $S(N) / \mathbf{m}^{k} S(N)$ is equal to that of the ring $S(N) / \mathbf{m} S(N)$. Now consider $R \subseteq S(N) \subseteq S(F)=$ polynomial ring in $\operatorname{rank}_{R}(F)$ variables over $R$. Applying the dimension inequality between $R$ and $S(N)$ gives $\operatorname{dim}(S(N)) \leq \operatorname{rank}_{R}(F)+d$. Since $\mathbf{m} \neq 0$ and $S(N)$ is a domain, it follows that $\operatorname{dim}(S(N) / \mathbf{m} S(N))$ is at most $\operatorname{rank}_{R}(F)+d-1$.

\section{BEHAVIOUR OF BUCHSBAUM-RIM MULTIPLICITY UNDER TRANSFORMS}

The goal of this section is to establish a basic inequality-Theorem 4.8concerning the Buchsbaum-Rim multiplicity of a module and those of its transforms. This inequality will be used to induce on the multiplicity to show that products of integrally closed modules are integrally closed.

In this section we will revert to the notation of Section 2 . So $R$ will be a twodimensional regular local ring with maximal ideal $\mathbf{m}$, infinite residue field $k$ and field of fractions $K$. Let $M$ be a finitely generated, torsion-free $R$-module and with (-)* denoting the functor $\operatorname{Hom}_{R}(-, R)$, let $F=M^{* *}$.

Recall that the module $M$ is said to be integrally closed if it is equal to its integral closure, $\bar{M}$, in the sense of Definition 3.1. Since $\bar{M} \subseteq M^{* *}$ over a Noetherian normal domain, $M$ is integrally closed precisely when each element of $F$ that is integral over $M$ is contained in $M$. Equivalently, if $S(M)$ denotes the image of the symmetric algebra $\operatorname{Sym}^{R}(M)$ in the polynomial ring $S(F)=\operatorname{Sym}^{R}(F)$, then $M$ is integrally closed when the first degree piece of the integral closure $\overline{S(M)}$ in $S(F)$ is equal to $M$.

The next proposition proves that integrally closed modules are contracted from rings of the form $R\left[\frac{\mathrm{m}}{x}\right]$ for $x \in \mathbf{m}-\mathbf{m}^{2}$. So all the results proved in Section 2 about contracted modules are applicable to integrally closed modules. This proposition is an intermediate step in proving that the transform of a integrally closed module is integrally closed.

In the proposition below and the rest of the paper, when we say that most $x \in \mathbf{m}$ satisfy a certain property we mean that the set of $x \in \mathbf{m}$ not satisfying the property is contained in a finite union of ideals each of which is strictly contained in $\mathbf{m}$. For instance, most $x \in \mathbf{m}$ are part of a minimal generating set of $\mathbf{m}$. Since the residue field of $R$ is assumed to be infinite, no ideal of $R$ is expressible as a finite union of ideals strictly contained in it. Thus, if most $x \in \mathbf{m}$ satisfy finitely many properties individually then most $x \in \mathbf{m}$ satisfy each of the properties.

Remark 4.1. For a Noetherian local ring $R$ with maximal ideal $\mathbf{m}$ and a discrete valuation ring $V$ with maximal ideal $\mathbf{n}$ that birationally dominates $R$, most $x \in \mathbf{m}$ generate $\mathbf{m} V$. For, since $\mathbf{m} V$ is principal, any element in $\mathbf{m} V-\mathbf{n m} V$ is a generator. Consider the ideal $\mathbf{n m} V \cap R$. This is an ideal of $R$ properly contained in $\mathbf{m}$ (since some element of $\mathbf{m}$ does generate $\mathbf{m} V$ ) and such that for any $x \in \mathbf{m}$ not in this ideal, $x$ generates $\mathbf{m} T$.

Remark 4.2. For a two-dimensional regular local ring $R$ with maximal ideal $\mathbf{m}$ and an m-primary ideal $I$ of $R$, for most $x \in \mathbf{m}, \operatorname{ord}_{R}(I)=\lambda_{R}(R /(I, x))$. 
Hence, for a torsion-free $R$-module $M$, if $M$ is contracted from $S=R\left[\frac{\mathrm{m}}{x}\right]$ for some $x \in \mathbf{m}-\mathbf{m}^{2}$, then $M$ is contracted from $S=R\left[\frac{\mathbf{m}}{x}\right]$ for most $x \in \mathbf{m}-\mathbf{m}^{2}$.

Proof. We always have an inequality $\operatorname{ord}_{R}(I) \leq \lambda_{R}(R /(I, x))$. Choose $a \in I$ of minimal order. By virtue of the inequality, it suffices to show that for most $x \in \mathbf{m}, \operatorname{ord}_{R}(a)=\lambda_{R}(R /(a, x))$. This will hold as long as the leading form of $x$ does not divide that of $a$ in the associated graded ring $\operatorname{gr}_{\mathrm{m}}(R)$ (which of course is a polynomial ring in two variables over the residue field of $R$ ). Consider the linear factors of the leading form of $a$ and lift them back arbitrarily to $R$. We get a finite set $x_{1}, x_{2}, \cdots, x_{k}$ of elements each of which is a minimal generator of $\mathbf{m}$. It is now easy to see that any $x \in \mathbf{m}$ avoiding the $k$ ideals $x_{i} R+\mathbf{m}^{2}$ cannot have leading form dividing that of $a$. This proves the first statement. The second follows from this and the proof of $(1) \Rightarrow(5)$ of Proposition 2.5.

Proposition 4.3. If $M$ is integrally closed, then for most $x \in \mathbf{m}$, we have that $M$ is contracted from $S=R\left[\frac{\mathrm{m}}{x}\right]$.

Proof. Since $M$ is integrally closed, by Definition 3.1 , we have that $M=$ $\bigcap M V$ where the intersection ranges over all the discrete valuation rings $V$ of $K$ that contain $R$. Since $F / M$ is of finite length, it is possible to choose finitely many such $V_{1}, V_{2}, \cdots, V_{n}$ such that $M=M V_{1} \cap M V_{2} \cap \cdots \cap M V_{n} \cap F$. Again, since $F / M$ is of finite length it vanishes on localization at any height 1 prime of $R$, and so it can be assumed that each of $V_{1}, V_{2}, \cdots, V_{n}$ is centered on $\mathbf{m}$.

Now, by the remarks above, most $x \in \mathbf{m}$ generate $\mathbf{m} V_{i}$ for each $i=$ $1,2, \cdots, n$. For any such $x$ then $\mathbf{m} / x \subseteq V_{i}$ for $i=1,2, \cdots, n$ and so $S=R\left[\frac{\mathrm{m}}{x}\right] \subseteq V_{i}$ for $i=1,2, \cdots, n$. Therefore $M S \cap F \subseteq M V_{1} \cap M V_{2} \cap \cdots \cap$ $M V_{n} \cap F=M$. This shows that $M$ is contracted from $S$.

The next proposition is a special case of the product theorem for integrally closed modules. The ideal $\mathbf{m}$ of $R$ is integrally closed and for a finitely generated torsion-free $R$-module $M$, the product $\mathrm{m} M$ in the sense of the tensor product $\mathbf{m} \otimes_{R} M$ modulo $R$-torsion is just the usual product $\mathbf{m} M$.

Proposition 4.4. If $M$ is integrally closed then so is $\mathbf{m} M$.

Proof. Since $F / M$ is of finite length, so is $F / \mathrm{m} M$ and then the characterization of the double dual in Proposition 2.1 shows that $(\mathbf{m} M)^{* *}$ is also equal to $F$. Therefore the module $N=\overline{\mathbf{m} M}$ is a submodule of $F$ and it is to be shown that $N=\mathbf{m} M$.

As in the proof of Proposition 4.3, choose discrete valuation rings $V_{1}, V_{2}, \cdots$, $V_{n}$ of $K$ birationally dominating $R$ such that both the equations $M=M V_{1} \cap$ $M V_{2} \cap \cdots \cap M V_{n} \cap F$ and $N=\mathbf{m} M V_{1} \cap \mathbf{m} M V_{2} \cap \cdots \cap \mathbf{m} M V_{n} \cap F$ hold. Then choose an $x \in \mathbf{m}$ such that $x V_{i}=\mathbf{m} V_{i}$ for each $i=1,2, \cdots, n$. For such an $x$ and any $v \in F$, if $x v \in N$ then $x v \in\left(\bigcap_{i=1}^{n} \mathbf{m} M V_{i}\right) \cap F=\left(\bigcap_{i=1}^{n} x M V_{i}\right) \cap F$. Hence $v \in\left(\bigcap_{i=1}^{n} M V_{i}\right) \cap F=M$ and this shows that $\left(N:_{F} x\right)=M$.

Suppose now that the submodule $\mathbf{m} M+x F$ of $F$ is integrally closed. Then $N=\overline{\mathbf{m} M} \subseteq \mathbf{m} M+x F$ and so $N=\mathbf{m} M+x\left(N:_{F} x\right)=\mathbf{m} M+x M=\mathbf{m} M$. This will complete the proof.

So it only needs to be seen that $\mathbf{m} M+x F$ is integrally closed. By a similar argument as the one used for $\mathbf{m} M$, it follows that $(\mathbf{m} M+x F)^{* *}=F$ and so 
to show that $\mathbf{m} M+x F$ is integrally closed we need to show that any element of $F$ integral over $\mathbf{m} M+x F$ is in $\mathbf{m} M+x F$. Consider such an element $v$. With $S(\mathbf{m} M+x F)$ denoting as usual the image of the image of the symmetric algebra of $\mathbf{m} M+x F$ in the symmetric algebra of $F$, the element $v$ satisfies an integral equation over $S(\mathbf{m} M+x F)$. Reducing this equation modulo $x$ gives an integral equation for the element $\widetilde{v} \in F / x F$ over the symmetric algebra of the image $\widetilde{\mathbf{m} M}$ of $\mathbf{m} M$ in $F / x F$. This shows that $\widetilde{v}$ is integral over the submodule $\widetilde{\mathrm{m} M}$ of the free module $F / x F$ over the discrete valuation ring $R / x R$. But, by definition of integral closure, any submodule of a free module over a discrete valuation ring is integrally closed. Thus $\widetilde{v} \in \widetilde{\mathbf{m M}}$. This implies that $v \in \mathbf{m} M+x F$ which is what is needed.

We next briefly recall the notion of quadratic transforms of a two-dimensional regular local ring and that of proper transform of an ideal.

A first quadratic transform of $R$ is a ring obtained by localizing a ring of the form $S=R\left[\frac{\mathrm{m}}{x}\right]$ at a maximal ideal containing $\mathrm{m} S$. For future use, we note that if $x, y$ generate $\mathbf{m}$, then any first quadratic transform of $R$ is obtainable as a localization of either $R\left[\frac{\mathrm{m}}{x}\right]$ or $R\left[\frac{\mathrm{m}}{y}\right]$. Such a ring is itself a two-dimensional regular local ring that birationally dominates $R$, i.e., it is a subring of $K$ containing $R$ and whose maximal ideal contains $\mathbf{m}$. We inductively define an $n^{\text {th }}$-quadratic transform of $R$ as a first quadratic transform of an $(n-1)^{\text {st }}$ quadratic transform of $R$. This again is a two-dimensional regular local ring which birationally dominates $R$. A quadratic transform (without qualification) of $R$ is an $n^{\text {th }}$-quadratic transform of $R$ for some $n$. We regard $R$ itself as a quadratic transform of $R$ with $n=0$.

By a standard result on quadratic transforms, if $T$ is a quadratic transform of $R$, there is then a unique sequence $R=T_{0} \subseteq T_{1} \subseteq \cdots T_{n-1} \subseteq T_{n}=T$ where each $T_{i+1}$ is a first quadratic transform of $T_{i}$ for $i=0,1, \cdots, n-1$. This result implies that under the relation of inclusion, the set of quadratic transforms of $R$ acquires a tree structure- $R$ itself on level 0 , the first quadratic transforms of $R$ on level 1 , the second quadratic transforms of $R$ on level 2 and so on.

For an m-primary ideal $I$ of $R$, the proper transform of the ideal in $S$ is defined to be the ideal $x^{-r} I S$ where $r=\operatorname{ord}_{R}(I)$. It is denoted by $I^{S}$. It is easy to see (and is shown in [Hnk]) that $S / I^{S}$ is an Artinian ring. For a first quadratic transform $T$ of $R$ the proper transform of $I$ in $T$ is denoted $I^{T}$ and defined to be $x^{-r} I T$. Note that if $T$ is a localization of $S$ then $I^{T}=I^{S} T$. For a quadratic transform $T$ of $R$ with associated sequence $R=T_{0} \subseteq T_{1} \subseteq$ $\cdots T_{n-1} \subseteq T_{n}=T$, the transform of $I$ in $T$ is defined inductively as $\left(I^{T}{ }\right)^{T}$. By convention the transform of $I$ in $R$ is set to be $I$ itself. The notion of the transform of an ideal is well-defined and it follows from the definition that if $T^{\prime}$ is a quadratic transform of $T$ and $T$ is a quadratic transform of $R$, then $I^{T^{\prime}}=\left(I^{T}\right)^{T^{\prime}}$. Note that the proper transform of an m-primary ideal of $R$ is primary to the maximal ideal of $T$ (or the whole of $T$ ).

The fundamental numerical result about complete ideals in two-dimensional regular local rings is the Hoskin-Deligne length formula for which several proofs are known. For a recent proof based on techniques of [Hnk] and references to other proofs, see [JhnVrm]. The theorem below is very much in the spirit of 
this formula but holds for general m-primary ideals instead of just complete ideals. The ideas in its proof can be easily extended to give another proof of the Hoskin-Deligne formula but we do not pause for that as it will not be needed in this paper.

Theorem 4.5. Let $I$ be an m-primary ideal of $R$ of order $r$ and $T$ be a first quadratic transform of $R$. Suppose that $\mathbf{m} T=x T$ for $x \in R$. Then the map

$$
\frac{\mathbf{m}^{r}}{I} \stackrel{x^{-r}}{\longrightarrow} \frac{T}{I^{T}}
$$

is a surjection of $R$-modules. In particular, $\lambda_{T}\left(T / I^{T}\right)<\lambda_{R}(R / I)$.

Proof. We have that $T$ is a localization of $S=R\left[\frac{\mathrm{m}}{x}\right]$ at one its maximal ideals. Further, the ring $S / I^{S}$ is Artinian and $T / I^{T}$ is one of its Artinian local factors. Therefore it suffices to see that the map

$$
\frac{\mathbf{m}^{r}}{I} \stackrel{x^{-r}}{\longrightarrow} \frac{S}{I^{S}}
$$

is surjective. Following this map up with multiplication by $x^{r}$ and using that $x^{r} S=\mathbf{m}^{r} S$ reduces the theorem to proving surjectivity of

$$
\frac{\mathbf{m}^{r}}{I} \rightarrow \frac{\mathbf{m}^{r} S}{I S}
$$

where the map is induced by inclusion. Therefore, we need to see that $\mathbf{m}^{r} S \subseteq$ $I S+\mathbf{m}^{r}$.

In proving this, there is no loss of generality in assuming that $I$ is contracted from $S$ since both $I$ and $I S \cap R$ have the same order and extend to the same ideal of $S$. Now,

$$
\operatorname{ord}_{R}(I)=r=\lambda_{R}\left(R /\left(\mathbf{m}^{r}, x\right)\right) \leq \lambda_{R}(R /(I, x))=\nu_{R}(I)-1
$$

the last equality from the proof of $(1) \Rightarrow(2) \Rightarrow(3)$ of Proposition 2.5. However, as the reverse inequality between $\operatorname{ord}_{R}(I)$ and $\nu_{R}(I)-1$ always holds by Proposition 2.2 , we have that there is equality and so $\left(\mathbf{m}^{r}, x\right)=(I, x)$. In particular, $\mathbf{m}^{r} \subseteq I+x \mathbf{m}^{r-1}$ and multiplying by $\mathbf{m}$ gives that $\mathbf{m}^{r+1} \subseteq I \mathbf{m}+x \mathbf{m}^{r}$.

With that as the basis case, an easy induction on $n$ gives $\mathbf{m}^{r+n} \subseteq I \mathbf{m}^{n}+x^{n} \mathbf{m}^{r}$ for all $n \geq 0$. Dividing this by $x^{n}$ shows that

$$
\mathbf{m}^{r} \cdot \frac{\mathbf{m}^{n}}{x^{n}} \subseteq I \cdot \frac{\mathbf{m}^{n}}{x^{n}}+\mathbf{m}^{r}
$$

Since $S$ is the union over all $n$ of $\frac{\mathrm{m}^{n}}{x^{n}}$, this completes the proof.

Proposition 4.6. Let $M$ be a integrally closed $R$-module and $T$ be a first quadratic transform of $R$. Then, the transform $M T$ is a integrally closed $T$ module.

Proof. Begin by choosing an $x \in \mathbf{m}-\mathbf{m}^{2}$ so that $T$ is a localization of $S=R\left[\frac{\mathbf{m}}{x}\right]$ at a maximal ideal containing $\mathrm{m} S$. Then $M T$ is a localization of $M S$ and so to show that $M T$ is integrally closed, it suffices by Corollary 3.3 to see that $M S$ is integrally closed.

We have $M S$ is contained in the free $S$-module $F S$ and since $S$ is an integrally closed domain, $F S$ is an integrally closed $S$-module. Hence, $\overline{M S} \subseteq$ $F S$. 
Consider an element $v \in F S$ integral over $M S$. By the equational criterion, the element $v \in \operatorname{Sym}_{1}^{S}(F S)$ satisfies an equation of the form $v^{n}+a_{1} v^{n-1}+\cdots+$ $a_{n}=0$ where the $a_{i} \in S(M S) \subseteq \operatorname{Sym}^{S}(F S)$. Since we are in the context of graded rings and homogeneous elements, we can further assume that $\operatorname{deg}\left(a_{i}\right)=i$ for each $i$. Now choose an integer $N$ so large that $x^{N} v \in F$ and such that for all $i, x^{N} a_{i} \in S(M) \subseteq \operatorname{Sym}^{R}(F)$.

Multiply the integral equation for $v$ with $x^{N n}$. Direct calculation shows that $a_{i} x^{N i} \in S\left(\mathbf{m}^{N} M\right) \subseteq \operatorname{Sym}^{R}(F)$. So we now have an integral equation for the element $x^{N} v$ over $S\left(\mathbf{m}^{N} M\right)$. By the equational criterion again, we have that $x^{N} v$ is integral over $\mathbf{m}^{N} M$. But by Proposition $4.4, \mathbf{m}^{N} M$ is integrally closed. Hence $x^{N} v \in \mathbf{m}^{N} M$. Dividing by $x^{N}$ gives that $v \in M S$ and completes the proof.

We will now analyze how the ideal $I(M)$ associated to a module $M$ behaves under transform. Recall that this ideal is the ideal of maximal minors of a matrix whose columns generate $M$ considered as a submodule of the free module $F$. The notation ()$^{*}$ in the proposition below refers to dualization with respect to the appropriate ring; $R$-modules are dualized with respect to $R$ and $T$-modules, with respect to $T$.

Proposition 4.7. Let $T$ be a quadratic transform of $R$. Then, $I(M T)=I(M)^{T}$. Proof. As observed after the proof of Proposition 2.2, the ideal $I(M)$ is an m-primary ideal of $R$ and similarly $I(M T)$ is an n-primary ideal of $T$ where $\mathbf{n}$ is the maximal ideal of $T$. The ideal $I(M)^{T}$ is also an n-primary ideal of $T$ and so to see that it is equal to $I(M T)$ it is enough to see that it is isomorphic to $I(M T)$.

Consider $M \subseteq F$ where $F$ is free and $\lambda_{R}(F / M)$ is finite. From here, we have $M T \subseteq F T$. Though $F T$ is a free $T$ module, in general $\lambda_{T}(F T / M T)$ is not finite. However we have $M T \subseteq(M T)^{* *} \subseteq F T$ and $M T$ is of finite colength in the free submodule $(M T)^{* *}$ of $F T$.

Choose a basis of $F$ and generators of $M$ and consider the following matrices. $\widetilde{D}$ has columns that are representations of the generators of $M$ with respect to the basis chosen for $F . \widetilde{C}$ is a square matrix whose columns are representations of a basis of $(M T)^{* *}$ with respect to the basis of $F T$ which is the same as that of $F . \widetilde{E}$ has columns whose columns which are representations of the generators of $M T$ with respect to the basis of $(M T)^{* *}$.

There is then a matrix equality $\widetilde{D}=\widetilde{C} \widetilde{E}$. By definition, $I(M)=$ ideal in $R$ of maximal minors of $\widetilde{D}$ and $I(M T)$ is the ideal in $T$ of maximal minors of $\widetilde{E}$. So from the matrix equation above, it follows that $I(M) T=\operatorname{det}(\widetilde{C}) \cdot I(M T)$. Therefore $I(M) T \cong I(M T) \Rightarrow I(M)^{T} \cong I(M T) \Rightarrow I(M)^{T}=I(M T)$.

We are now ready to prove the main result of this section. We will show that the Buchsbaum-Rim multiplicity $e\left(M^{* *} / M\right)$ associated to a finitely generated torsion-free module $M$ decreases on taking transforms. The main point of the proof is to express the Buchsbaum-Rim multiplicity in terms of the colength of a certain ideal and then to use Theorem 4.5 to ensure its decrease.

Theorem 4.8. Let $T$ be a first quadratic transform of $R$ and let $M$ be a finitely generated torsion-free $R$-module. Then $e\left((M T)^{* *} / M T\right)<e\left(M^{* *} / M\right)$. 
Proof. Consider $M \subseteq M^{* *}=F$ with $F$ free and with $\lambda_{R}(F / M)$ finite. As usual, we denote $\operatorname{rank}_{R}(F)$ by $r$. By Theorem 3.4, there is a submodule $N \subseteq M$ such that $N$ is generated by $r+2-1=r+1$ elements and is a reduction of $M$, i.e., every element of $M$ is integral over $N$.

From the determinantal criterion, it follows that the ideal of maximal minors of generators of $N$ is a reduction of $I(M)$ and in particular m-primary. Since this ideal multiplies $F$ into $N$, we have that $N$ is of finite colength in $F$ and $F=N^{* *}$ by Proposition 2.1 .

Since $N$ is a reduction of $M$ we have by Proposition 3.8 that $e(F / M)=$ $e(F / N)$ and since $N$ is generated by $\operatorname{rank}_{R}(F)+2-1$ elements over a CohenMacaulay ring we have by Proposition 3.7 that $e(F / N)=\lambda_{R}(F / N)$.

In some basis of $F$, suppose that $N$ is generated minimally by the columns of the $r \times(r+1)$ matrix $\tilde{N}$. The ideal of maximal minors of $\widetilde{N}$ is by definition $I(N)$ and is m-primary. So by the Hilbert-Burch theorem, $R / I(N)$ has a resolution,

$$
0 \longrightarrow F^{*} \stackrel{\widetilde{N}^{*}}{\longrightarrow} G^{*} \longrightarrow R \longrightarrow 0
$$

where $\operatorname{rank}_{R}(G)=r+1$. Dualizing this resolution shows that $\operatorname{Ext}_{R}^{2}(R / I(N), R)$ $\cong F / N$. But by local duality ([BrnHrz, Theorem 3.5.8]), $\operatorname{Ext}_{R}^{2}(R / I(N), R) \cong$ $(R / I(N))^{2}$-the Matlis dual of the module $R / I(N)$. So

$$
\lambda_{R}(R / I(N))=\lambda_{R}\left((R / I(N))^{\smile}\right)=\lambda_{R}\left(\operatorname{Ext}_{R}^{2}(R / I(N), R)\right)=\lambda_{R}(F / N) .
$$

Summarizing, we have so far shown that for any $r+1$ generated reduction $N$ of $M, e\left(M^{* *} / M\right)=\lambda_{R}(R / I(N))$.

Next we consider the quadratic transform $T$ of $R$. The inclusion of $R$ modules $N \subseteq M$ gives an inclusion of $T$-modules $N T \subseteq M T$. Since $N$ is a reduction of $M$, it follows from the equational criterion that $N T$ is a reduction of $M T$. Also $N T$ is generated by $r+1=\operatorname{rank}_{T}(M T)+1$ elements. Therefore, $e\left((M T)^{* *} / M T\right)=\lambda_{T}(T / I(N T))$.

Now, by Proposition 4.7, $I(N T)=I(N)^{T}$ and then by Theorem 4.5, $\lambda_{T}\left(T / I(N)^{T}\right)<\lambda_{R}(R / I(N))$. Hence,

$e\left((M T)^{* *} / M T\right)=\lambda_{T}(T / I(N T))=\lambda_{T}\left(T / I(N)^{T}\right)<\lambda_{R}(R / I(N))=e\left(M^{* *} / M\right)$.

This finishes the proof.

\section{INTEGRAL CLOSURE OF PRODUCTS, SYMMETRIC POWERS AND EXTERIOR POWERS}

In this last section we prove the main results of this paper and work out an example. The main results all assert that some module or ideal is integrally closed and can all be regarded as generalizations of Zariski's product theorem. We then show the non-triviality of the theory by giving a general construction of integrally closed indecomposable modules. Based on this construction, we exhibit an explicit example of such a module.

The notations in this section are the same as those of Section 4 . Thus, $R$ will be a two-dimensional regular local ring with maximal ideal $\mathbf{m}$, infinite residue field $k$ and field of fractions $K . M$ will be a finitely generated torsion-free $R$-module and $F$ will denote its double dual $M^{* *}$. 
Proposition 5.1. For $x \in \mathbf{m}-\mathbf{m}^{2}$ let $S=R\left[\frac{\mathrm{m}}{x}\right]$. Then, $M S=F S \cap(\bigcap M T)$ where the second intersection ranges over all the first quadratic transforms $T$ of $R$ that are localizations of $S$.

Proof. It is clear that $M S \subseteq F S$ and that $M S \subseteq M T$ for each $T$ as in the proposition. To show the reverse inclusion, note that $M S=\bigcap(M S)_{\mathbf{n}}$ where the intersection ranges over all the maximal ideals $\mathbf{n}$ of $S$ where we identify $(M S)_{\mathbf{n}}$ with the submodule $M S_{\mathbf{n}}$ of $M_{K}$. Now consider an element $v \in$ $F S \cap(\bigcap M T)$. We will show that $v \in M S$ by showing that $v \in M S_{\mathbf{n}}$ for every maximal ideal $\mathbf{n}$ of $S$. There are two cases to consider. Case 1: $\mathbf{m} S \subseteq \mathbf{n}$. In this case the localization $S_{\mathrm{n}}$ is, by definition, a quadratic transform $T$ of $R$ and therefore $v \in M S_{\mathbf{n}}$. Case 2: $\mathbf{m} S \notin \mathbf{n}$. In this case, the ideal $\mathbf{n}$ is of height 1 and has height 1 contraction to $R$. Since $F / M$ is of finite length, $F_{P}=M_{P}$ for any height 1 prime $P$ of $R$ and therefore $M S_{\mathrm{n}}=F S_{\mathrm{n}}$. Hence $v \in F S \subseteq F S_{\mathbf{n}}=M S_{\mathbf{n}}$. This completes the proof.

Theorem 5.2. Suppose that $M$ and $N$ are finitely generated torsion-free integrally closed $R$-modules. Then $M N$ is integrally closed.

Proof. Set $F=M^{* *}$ and $G=N^{* *}$. Then $F$ and $G$ are free $R$-modules containing $M$ and $N$ respectively and with finite length quotients. We will prove that $M N$ is integrally closed by induction on $\min \{e(F / M), e(G / N)\}$.

If this minimum is 0 , then one of $M$ or $N$ is free and then $M N$ is just a finite direct sum of copies of the other. Since a direct sum of integrally closed modules is integrally closed, we are done in this case.

Suppose the minimum is positive. By Proposition 4.3, choose an $x \in \mathbf{m}-\mathbf{m}^{2}$ such that both $M$ and $N$ are contracted from $S=R\left[\frac{\mathrm{m}}{x}\right]$. By Theorem 2.8 we have that $M N$ is also contracted from $S$. Thus, if $H=F \otimes_{R} G$, then $H=(M N)^{* *}$ and $M N=M N S \cap H \subseteq H S$. Suppose now that $M N S$ is shown to be an integrally closed $S$-module. Then, consider any element of $H$ integral over $M N$. This element is also integral over $M N S$ and hence is in $M N S$. Therefore the element is in $M N$. This will show that $M N$ is integrally closed and complete the proof.

We now show that $M N S$ is integrally closed. Since $S$ is an integrally closed domain, by Corollary 3.3 , it is enough to verify that for any maximal ideal $\mathbf{n}$ of $S, M N S_{\mathrm{n}}$ is an integrally closed $S_{\mathrm{n}}$-module.

There are two cases to consider. If $\mathbf{m} S \notin \mathbf{n}$, then $\mathbf{n}$ is of height 1 and so $S_{\mathrm{n}}$ is a discrete valuation ring over which any module is integrally closed. If $\mathbf{m} S \subseteq \mathbf{n}$, then $S_{\mathbf{n}}$ is a first quadratic transform $T$ of $R$ and here we have to see that $M N T$ is integrally closed.

By definition of the product and transform operations, $M N T=M T . N T$. We have by Proposition 4.6 that each of $M T$ and $N T$ is integrally closed. Then, by Theorem 4.8,

$$
\min \left\{e\left((M T)^{* *} / M T\right), e\left((N T)^{* *} / N T\right)\right\}<\min \{e(F / M), e(G / N)\} .
$$

So by the inductive hypothesis $M T . N T$ is integrally closed which finishes the proof.

Theorem 5.3. Suppose that $M$ is a finitely generated torsion-free integrally closed $R$-module. Then the torsion-free symmetric algebra $S(M)$ is a Noetherian normal domain. 
Proof. Since $S(M)$ is a graded domain, its integral closure is also graded and its graded pieces are the the integral closures of the modules $S_{n}(M)$. Thus, to see that $S(M)$ is a normal domain, it suffices to see that each $S_{n}(M)$ is an integrally closed module. For this, we follow the ideas of the previous proof.

Set $F=M^{* *}$ and induce on the Buchsbaum-Rim multiplicity of $F / M$, the case when it is zero being trivial. Otherwise, by Proposition 4.3, choose an $x \in \mathbf{m}$ so that $M$ is contracted from $S=R\left[\frac{\mathrm{m}}{x}\right]$. By Theorem 2.8 all the $S_{n}(M)$ are also contracted from $S$ and so to show that they are integrally closed it suffices to show that all $S_{n}(M) S$ are integrally closed.

Using Corollary 3.3 as in the previous proof, we reduce to showing that for a first quadratic transform $T$ of $R$, all the $S_{n}(M) T$ are integrally closed. But by definitions, $S_{n}(M) T=S_{n}(M T)$, which are integrally closed since $M T$ is integrally closed by Proposition 4.6 and by the inductive assumption. This finishes the proof.

The proof of the following theorem follows by essentially the same reasoning applied to the modules $E_{n}(M)$.

Theorem 5.4. Suppose that $M$ is a finitely generated torsion-free integrally closed $R$-module. Then each $E_{n}(M)$ is integrally closed. In particular, the ideal of maximal minors $I(M)$ is integrally closed. Furthermore, taking integral closure commutes with taking the ideal of maximal minors for any finitely generated torsion-free R-module.

Proof. Set $F=M^{* *}$ and induce on the Buchsbaum-Rim multiplicity of $F / M$, the case when it is zero being trivial. Otherwise, by Proposition 4.3, choose an $x \in \mathrm{m}$ so that $M$ is contracted from $S=R\left[\frac{\mathrm{m}}{x}\right]$. By Theorem $2.8 E_{n}(M)$ is also contracted from $S$ and so to show that it is integrally closed it suffices to show that its transform in $S$ is integrally closed.

By the local-global principle, it is enough to see that for a quadratic transform $T$ of $R$, the module $E_{n}(M) T$ is integrally closed. But by definitions, $E_{n}(M) T=E_{n}(M T)$, which is integrally closed since $M T$ is integrally closed by Proposition 4.6 and by the inductive assumption.

Since $I(M)$ is the same as $E_{r}(M)$ where $r=\operatorname{rank}_{R}(M)$, it is integrally closed. For an arbitrary finitely generated $R$-module $M$, we have by the determinantal criterion that $I(\bar{M})$ is integral over $I(M)$ and then by the previous line that $I(\bar{M})$ is integrally closed-it follows that $I(\bar{M})=\overline{I(M)}$.

Remark 5.5. The theorem above shows that the first Fitting ideal of $F / M$ (or equivalently by the proof of Proposition 2.2, the first Fitting ideal of $M$ ) is integrally closed if $M$ is. However, it is not true that the other Fitting ideals of $F / M$ are integrally closed. For instance, if $M$ is the direct sum of ideals $I=\left(x^{2}, x y^{3}, y^{5}\right)$ and $J=\left(y^{3}, y^{2} x^{2}, y x^{3}, x^{4}\right)$, then $M$ is integrally closed since each of the ideals is, but the second Fitting ideal of $F / M$ is the sum of the ideals $I+J=\left(x^{2}, y^{3}\right)$ which is not integrally closed. For an arbitrary integrally closed module $M$, I do not know whether all Fitting ideals of $M$ itself are integrally closed.

Remark 5.6. We point out that while we have proved and used the reduction of Buchsbaum-Rim multiplicity theorem, a different approach to the proof of the main theorems is to induce on $\lambda_{R}(R / I(M))$. That this also decreases under 
transforms follows from Theorem 4.5 and Proposition 4.7. Still, Theorem 4.8 is interesting in itself and we hope to show in a future paper an equality for the Buchsbaum-Rim multiplicity analogous to the formula

$$
e(I)=\sum \operatorname{ord}_{T}\left(I^{T}\right)^{2}
$$

that holds for an m-primary ideal of $R$ (assuming that the residue field is algebraically closed). In the formula, the sum is over all quadratic transforms $T$ of $R$ and this obviously implies that for any such $T, e\left(I^{T}\right)<e(I)$.

In Example 3.5, we considered an indecomposable $R$-module $M$ and found that its integral closure $N$ was a direct sum of ideals $\mathbf{m} \oplus \mathbf{m}$. If it were to be true that every integrally closed module decomposed as a direct sum of ideals, then Theorem 5.2 would follow trivially from Zariski's product theorem. In the theorem below we show that this is not the case by showing how to construct indecomposable integrally closed modules over any two-dimensional regular local ring.

We require the notion of a simple integrally closed ideal of $R$. This is a integrally closed ideal of $R$ which cannot be expressed a product of strictly larger proper ideals.

Theorem 5.7. Let $N$ be an $R$-module without a free direct summand such that $\overline{I(N)}$ is simple. Then $\bar{N}$ is an indecomposable R-module. In particular, there exist indecomposable integrally closed $R$-modules of arbitrary rank.

Proof. Note that a torsion-free $R$-module $N$ has no free direct summand exactly when $N \subseteq \mathrm{m} F$ where $F=N^{* *}$. Since $\mathrm{m} F$ is integrally closed, it follows that $N$ has a free direct summand if and only if $\bar{N}$ does.

Suppose by way of contradiction that $\bar{N}=P \oplus Q$ is a non-trivial decomposition. Then $I(\bar{N})=I(P) \cdot I(Q)$. Since $I(\bar{N})=\overline{I(N)}$ is simple by assumption, at least one of $I(P)$ or $I(Q)$ is $R$ which means that at least one of $P$ or $Q$ is free. This contradicts the assumption that $\bar{N}$ has no free direct summand.

To construct modules that do not have a free summand and whose ideal of minors has simple integral closure, begin with any ideal $J$ whose integral closure is simple and resolve $R / J$ minimally as below.

$$
0 \longrightarrow F \stackrel{\phi}{\longrightarrow} G \stackrel{J}{\longrightarrow} R \longrightarrow 0
$$

Then, consider the transpose map $G^{*} \stackrel{\phi^{*}}{\longrightarrow} F^{*}$. Its image has no free summand by minimality and has ideal of minors $J$. Taking, for instance, $J$ to be the integral closure of $\left(x^{r}, y^{r+1}\right)$, where $x$ and $y$ form a regular system of parameters of $R$, produces a module of rank $r$.

To conclude, we use the theorem to give an explicit example of a integrally closed indecomposable $R$-module.

Example 5.8. Let $R=k[[x, y]]$ be a ring of formal power series over a field $k$. Denote by $F$ the free $R$-module $R \oplus R$ of rank 2 whose elements are column matrices with two entries. The ideal $I=\left(y^{3},-x y^{2}, x^{2}\right)$ is a simple integrally closed ideal of $R$ and $R / I$ is minimally resolved by

$$
0 \longrightarrow R^{2} \stackrel{\phi}{\longrightarrow} R^{3} \longrightarrow R \longrightarrow 0
$$


where $\phi$ has a matrix representation as

$$
\left(\begin{array}{cc}
x & 0 \\
y & x \\
0 & y^{2}
\end{array}\right)
$$

In the notation of the Theorem 5.7, N is the submodule of $R^{2}$ (actually of $\left.\left(R^{2}\right)^{*}\right)$ generated by the columns of the matrix

$$
\left(\begin{array}{ccc}
x & y & 0 \\
0 & x & y^{2}
\end{array}\right)
$$

We will show that $\bar{N}$ is the submodule of $R^{2}$ generated by the columns of the matrix

$$
\left(\begin{array}{cccc}
x & y & 0 & 0 \\
0 & x & y^{2} & x y
\end{array}\right)
$$

Let $T$ be the column vector $\left[\begin{array}{ll}1 & 0\end{array}\right]^{t}$ and $U$ be the column vector $\left[\begin{array}{ll}0 & 1\end{array}\right]^{t}$. By using any of the three criteria as in Example 3.5, it is easily seen that $x y U$ is integral over $N$. So we next need to see any element integral over $N$ is in the submodule, say $M$, of $F$ generated by $x T, y T+x U, y^{2} U$ and $x y U$. Equivalently, we want to see that $M$ is integrally closed.

To see this, note that by (4) and (5) of Proposition 2.5, $M$ is contracted from $S=R\left[\frac{\mathrm{m}}{y}\right]$ and so to see that $M$ is integrally closed it suffices to see that its transform $M S$ is integrally closed. By row and column operations over $S$, it is easy to see that $M S \cong S \oplus P$ where $P$ is the maximal ideal $(y, x / y) S$.

The only localization of $S$ at which $S \oplus P$ is not actually free is at $P$. Denote by $T$ the first quadratic transform $S_{P}$ of $R$ and let $\mathbf{n}$ be its maximal ideal. Then $(S \oplus P)_{P} \cong T \oplus \mathbf{n}$ which is clearly an integrally closed $T$-module. Therefore $S \oplus P$ and hence $M$ is integrally closed.

Thus $M$ is an integrally closed indecomposable $R$-module. It seems too optimistic to expect that a converse to Theorem 5.7 hold in the sense that an indecomposable complete $R$-module of rank bigger than 1 have a simple complete ideal of maximal minors but it would be nice to have this clarified with either a proof or counterexample.

Remark 5.9. Professor Lipman has pointed out that Theorems 5.2 and 5.3 also hold for two-dimensional rings with rational singularities. With the appropriate definitions of integrally closed sheafs of modules, the proofs can be deduced by generalizing the proofs of Theorems 7.1 and 7.2 of [Lpm2]. Alhough these theorems are stated and proved for sheafs of ideals, one of the crucial technical facts ([Lpm, Lemma 7.3]) is proved there for modules and is the key to generalization. It would be interesting to know whether the numerical relationships for integrally closed modules such as $\nu_{R}(M) \leq \operatorname{rank}_{R}(M)+\operatorname{ord}_{R}(M)$ and the decrease of Buchsbaum-Rim multiplicity on transforms also generalize.

\section{ACKNOWLEDGMENTS}

The material in this paper formed part of my doctoral thesis directed by Professor Bill Heinzer. I am deeply grateful to him for his guidance and encouragement. I would also like to thank the other members of my thesis committeeProfessors Luchezar Avramov, Craig Huneke and Joseph Lipman-for their 
input and comments. My thanks to Radha Mohan and to Dan Katz for their encouragement and several suggestions for improvement during the rather long gestation period of this paper.

\section{REFERENCES}

[BrnHrz] W. Bruns and J. Herzog, Cohen-Macaulay rings, Cambridge Stud. Adv. Math., 39, Cambridge University Press, 1993.

[BchSnb] D. A. Buchsbaum and D. Eisenbud, Some structure theorems for finite free resolutions, Adv. Math. 12 (1974), 84-139.

[BchRm] D. A. Buchsbaum and D. S. Rim, A generalized Koszul complex II. Depth and multiplicity, Trans. Amer. Math. Soc. 111 (1964), 197-224.

[Ctk] S. D. Cutkosky, Factorization of complete ideals, J. Algebra 115 (1988), 151-204.

[Ctk2] _ Complete ideals in algebra and geometry, Commutative Algebra: Syzygies, Multiplicities and Birational Algebra, Contemporary Math., vol. 159, Amer. Math. Soc., Providence, RI, 1993, pp. 27-39.

[Ghn] H. Gohner, Semifactoriality and Muhly's condition (N) in two-dimensional local rings, J. Algebra 34 (1975), 403-429.

[Hnk] C. Huneke, Complete ideals in two dimensional regular local rings, Commutative Algebra, Proceedings of a Microprogram, MSRI publ. no. 15, Springer-Verlag, 1989, pp. 417-436.

[Hnk2] - The primary components of and integral closures of ideals in three-dimensional regular local rings, Math. Ann. 275 (1986), 617-635.

[HnkSII] C. Huneke and J. Sally, Birational extensions in dimension two and integrally closed ideals, J. Algebra 115 (1988), 481-500.

[JhnVrm] B. Johnston and J. K. Verma, On the length formula of Hoskin and Deligne and associated graded rings of two-dimensional regular local rings, Math. Proc. Cambridge Philos. Soc. 111 (1992), 423-432.

[Ktz] D. Katz, Reduction criteria for modules, Preprint.

[KlmThr] S. Kleiman and A. Thorup, A geometric theory of the Buchsbaum-Rim multiplicity, J. Algebra 167 (1994), 168-231.

[Krb] D. Kirby, On the Buchsbaum-Rim multiplicity associated with a matrix, J. London Math. Soc. (2) 32 (1985), 57-61.

[KrbRs] D. Kirby and D. Rees, Hilbert functions of multigraded modules and the Buchsbaum-Rim multiplicity.

[Lpm] J. Lipman, On complete ideals in regular local rings, Algebraic Geometry and Commutative Algebra in honor of Masayoshi Nagata, Academic Press, 1987, pp. 203-231.

[Lpm2] _ Rational singularities with applications to algebraic surfaces and unique factorization, Publ. Math. Inst. Hautes Etudes Sci. 36 (1969), 195-279.

[Rs] D. Rees, Reduction of modules, Math. Proc. Cambridge Philos. Soc. 101 (1987), 431-449.

[ZrsSml] O. Zariski and P. Samuel, Commutative algebra, Vol. II, Van Nostrand Reinhold, New York, 1960.

Department of Mathematics, University of Kansas, Lawrence, Kansas 66045

E-mail address: kamala@oberon.math.ukans.edu 